\title{
Application of microbial technology to nanomaterial patches
}

\author{
Rui Su \\ University of California, Berkeley, 94720, PRC \\ rui999@berkeley.edu
}

\begin{abstract}
Microbial technology has been widely used in various industries, to study the application of microbial technology in nanomaterial patches. The nanotube arrays were synthesized by microbial technology, and the nanopatch particles were further prepared with a JEM-2010 transmission electron microscope (Joep, Japan) operating at a voltage set to $200 \mathrm{kV}$.Test the performance of nanomaterials with application of microbial technology in nanomaterial patches according to the results of the electrocatalytic activity test, $\mathrm{Au}-\mathrm{TiO} 2$ nanocomposites have obvious electrocatalytic activity in oxygen reduction, develop the photocatalytic performance test, further study the photocatalytic activity of nanocomposites with different $\mathrm{Au}$ loading,The activity order was concluded to be Au-TiO2 (4.5\%) > $\mathrm{Au}-\mathrm{TiO} 2(3.4 \%)>\mathrm{Au}-\mathrm{TiO} 2(1.4 \%)>\mathrm{Au}-\mathrm{TiO} 2(6.4 \%)>\mathrm{TiO} 2$.
\end{abstract}

\section{CCS CONCEPTS}

- General and reference $\rightarrow$ Cross-computing tools and techniques.

\section{KEYWORDS}

Nanomaterial, Microbial technology, Activity order

\section{ACM Reference Format:}

Rui Su. 2021. Application of microbial technology to nanomaterial patches. In 2021 10th International Conference on Bioinformatics and Biomedical Science (ICBBS 2021), October 29-31, 2021, Xiamen, China. ACM, New York, NY, USA, 4 pages. https://doi.org/10.1145/3498731.3498763

\section{INTRODUCTION}

In recent years, there has been an increasing range of applications of microbial technology due to the fact that in itself there are advantages of enhanced biological activity that are able to bring it to the forefront of both medicine and industry.At present, at home and abroad for the application of microbial technology in various industries, extensive research is unfolded, obtained that the composition of reducing substances inside microbial technology is complex, may contain a variety of reducing substances, some scholars conclude that can adopt this method to synthesize nanomaterials, its advantage is green synthesis, and microorganisms are easy to culture, fast reproduction.Therefore, in this paper, we tried to apply microbial technology in nanomaterial patches, and endeavored to improve the performance of nanomaterial patches and create a new field of research for the development of microbial technology.

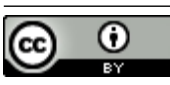

This work is licensed under a Creative Commons Attribution International 4.0 License.

ICBBS 2021, October 29-31, 2021, Xiamen, China

(C) 2021 Copyright held by the owner/author(s).

ACM ISBN 978-1-4503-8430-8/21/10.

https://doi.org/10.1145/3498731.3498763

\section{TRIAL PREPARATION}

\subsection{Nanotube array synthesis by microbial technology}

The synthesis of nanomaterials using microbial technology is characterized by a large area of synthesized nanomaterials, and better catalytic performance, which can reduce production energy consumption to a certain extent, catalytically reduce compounds while reducing environmental pollution, and have high-strength stability.A variety of synthetic methods have been developed to prepare the novel nanofibrous structured material $\mathrm{TiO} 2$, such as oxidation synthesis placed in the anode, template method, hydrothermal rubber synthesis reaction, sol-solidified rubber synthesis, and vapor phase solid deposition synthesis in physicochemical.Among them, $\mathrm{TiO} 2$ nanodiode integrated array device produced by the process of nanoanode tube and alumina method has a simple structure with various morphologies.It is easy to control and enables strong binding to plates.The bi-directional wide band gap and large probability application of high-density electron hole material composite of $\mathrm{TiO} 2$ nanomaterial greatly reduce its efficiency in using photocatalysts, which must be modified to improve its photocatalytic performance.By analytically adjusting the parameters of various anode preparers (including electrolyte composition, anodic heated oxidation reaction voltage, anodic heated oxidation reaction time, anodic heated oxidation reaction temperature, etc.), it was investigated that the parameters of various anode preparers did not affect the overall morphology of $\mathrm{TiO} 2$ nanocrystal anode arrays fabricated by microbial technology.By using the overall photocatalytic electronic decomposition performance of Alizarin Red phosphate solution to study the overall photocatalytic decomposition performance of nano supermicrotubule molecular arrays with different molecular morphology types of $\mathrm{TiO} 2$, we find a relatively optimal morphology for the photocatalytic decomposition performance and the relative specific preparation process conditions of $\mathrm{TiO} 2$ nano supermicrotubule molecular arrays [1].Based on the above discussion, the smaller nanotube density and bamboo node resulted in the generation of longer nanotubes at $40 \mathrm{~V}$ anodization voltage, nanotubes prepared at different voltages in electrolyte with a propanol / water volume ratio of 4:1 and NH4F concentration, as shown in Table 1

Based on these results, the $\mathrm{TiO} 2$ nanotube arrays were modified by $\mathrm{Zr}$ doping and $\mathrm{TiO} 2$ / $\mathrm{ZnO}$ Composite.The main electrochemical method for the fabrication of electrode arrays for TiO2 nanotrystals is the oxidation system using a two ion electrode with titanium oxide sheets as anode, a Pt electrode as cathode, and an electrolyte of glycerol and water containing $0.5 \mathrm{wt} \%$ ammonium fluoride at a set distance of $4 \mathrm{~cm}$.A DC power supply was used to apply voltage to the two electrode system at a rate of $0.1 \mathrm{~V} / \mathrm{sec}$, and experiments were performed under a magnetic stirrer. 
Table 1: Parameters of 1tio2nanotube arrays

\begin{tabular}{llll}
\hline Voltage/V & 20 & 40 & 60 \\
\hline Tubelength/nm & 1.25 & 6.74 & 4.82 \\
Pipediameter/nm & 110 & 185 & 270 \\
Spacenode/nm & 56 & 56 & 56 \\
\hline
\end{tabular}

\subsection{Preparation of nanopatch particles}

Nanoparticle preparation was prepared by reducing ruthenium chloride in microorganisms to ethylene glycol. Briefly, $82 \mathrm{mg}$ of sodium acetate and $50 \mathrm{mg}$ of ruthenium chloride were added to 100 $\mathrm{ml}$ of 1,2-ethylene glycol (20 $\mathrm{min}$ in advance). The mixture was refluxed at $165^{\circ} \mathrm{C}$ for $1 \mathrm{~h}$. After cooling to room temperature, $81.2 \mathrm{mg}$ of 5-hexenoic acid or $66.7 \mathrm{mg}$ of 6-mercaptoacetic acid was added in a round bottom flask and stirred for 2 days. The product was then placed in a dialysis membrane and dialyzed against deionized water for 2 days. The first day the water was changed every $30 \mathrm{~min}$ and the following day the water change interval was extended as appropriate. After dialysis, the product in the dialysis membrane was centrifuged and washed with methanol several times. The preparation of ru-tio2 nanoparticles was similar to the method previously used for the preparation of TiO2.0.1 ml m-chloroaniline and $\mathrm{Ru}$ nanoparticles containing $1 \mathrm{mg}$ different ligands were added to $10 \mathrm{ml}$ toluene and then transferred into the liner of a hot pot [2]. Next, $0.17 \mathrm{~g}$ tetrabutyl titanate was added into $10 \mathrm{ml}$ deionized water and transferred into the liner of a hot pot. The mixed solution was hydrothermally reacted at $180^{\circ} \mathrm{C}$ for $12 \mathrm{~h}$. The resulting grey product was then centrifuged and washed with methanol several times. The products were represented by ru-h-tio2 (prepared with 5-hexanoic acid) and ru-m-tio2 (prepared with 6-mercaptoacetic acid).

\subsection{Selection of specimens}

A JEM-2010 transmission electron microscope test at Joep, Japan, operating at $200 \mathrm{kV}$ was employed. The sample was dispersed in toluene and dropped onto a copper grid. The phases of the samples were determined by a RIGAKU small powder diffractometer using a Cuk $\alpha$ Radiation characterization, testing angle ranged from $2 \theta=20$ ${ }^{\circ}-70^{\circ}$ at a testing rate of $5 \%$ min. The UV Vis absorption spectra of the specimens were tested with an ati-unicamuv4 spectrometer. Fluorescence spectra were tested by PTI photoluminescence spectrophotometer. X-ray photoelectron spectra were characterized by a phi5400xps instrument using ALK $\alpha$ Radiation at a power of 350 $\mathrm{W}$ and a pressure of 10-9 Torr. The functional groups on the surface of the specimens were analyzed by Fourier transform infrared (FTIR) spectroscopy in the experiment. Both samples showed severe agglomeration (A and $\mathrm{b})$.The average diameters of the samples were all around $5 \mathrm{~nm}$ [3].HRTEM images indicated clear lattice fringes in both samples with approximate lattice spacing of 0.205 $\mathrm{nm}, 0.350 \mathrm{~nm}$, and $0.380 \mathrm{~nm}$ (red line), corresponding to the (101) crystal plane 181 of $\mathrm{Ru}$, and the (101) and (100) crystal planes of $\mathrm{TiO} 2$, respectively.

\section{NANOMATERIAL PERFORMANCE TESTING}

\subsection{Electrocatalytic activity test}

Electrocatalytic activity test the electrocatalytic activity of $\mathrm{Au}-\mathrm{TiO} 2$ nanocomposites prepared in this paper by microbial technique in oxygen reduction reaction was further examined by voltammetry, setting an oxygen saturation of $0.1 \mathrm{~m}$ in $\mathrm{NaOH}$ solution. Different electrode rotation rates were taken in the range of $100 \mathrm{rpm}-2000$ rpm, the solutions were oxygen saturated 0.1 mnaoh solutions, the rate of the electrode potential sweep reached $10 \mathrm{MV} / \mathrm{s}$ on average, and four different sets of sample parameters were determined at (a) $\mathrm{TiO} 2$ (b) Tio2-Au (1.4\%); (c) Tio2-Au (4.5\%); (d) The test was unfolded under $\mathrm{TiO} 2 \mathrm{Au}(6.4 \%)$, and the test results are shown in Figure 1

Observing the 4 sets of test results in Fig. 1 above, it was found that the catalytic performance of $\mathrm{Au}-\mathrm{TiO} 2$ nanocomposites with different $\mathrm{Au}$ loadings also varied. The $\mathrm{Au}-\mathrm{TiO} 2$ nanocomposite with an $\mathrm{Au} / \mathrm{Ti}$ atomic ratio of $4.5 \%$ exhibited the highest limiting current within the existing experimental range, indicating its strongest catalytic ability in oxygen reduction. The improved catalytic activity of $\mathrm{TiO} 2 \mathrm{Au}(\mathrm{x})$ nanocomposites can be attributed to the strong metal support interaction, with the produced Ti (III) serving as the active center for catalysis, thus leading to the decomposition of water and the formation of surface adsorbed hydroxyl moieties. In the test, when the electrode potential was scanned to $+0.8 \mathrm{~V}$, the cathodic current began to appear in all samples, and the current began to stabilize when the electrode potential reached to +0.4 $\mathrm{V}$, which illustrated that $\mathrm{Au}-\mathrm{TiO} 2$ nanocomposites had an obvious electrocatalytic activity in oxygen reduction.

\subsection{Photocatalytic performance test}

Applications photocatalytic activity of $\mathrm{Zr}$ doped molecular $\mathrm{TiO} 2$ nanotransducers molecular arrays were characterized by the application photocatalysts and degradation reactions of Alizarin Red molecular solution under highly ultraviolet and strong irradiation conditions. This refers to different photocatalytic rates and degradation rates to a series of Alizarin infrared solutions with different values of $\mathrm{Zr}$ doped $\mathrm{TiO} 2$ nanotransducers that can be prepared at different concentrations and voltages in different halogen electrolytes. The analysis showed that the chemical photocatalytic and degradation rates for Alizarin Red molecular solution were significantly higher than those for pure $\mathrm{TiO} 2$ nanomicrotubule molecular arrays (39.20\%) doped in all Zr plots [4].For $\mathrm{Zr}$ doped $\mathrm{TiO} 2$ nanotube arrays prepared with electrodeposition voltage of $3 \mathrm{~V}, 6 \mathrm{~V}$ and $9 \mathrm{~V}$, the photocatalytic degradation rate of the samples increased with increasing electrolysis concentration. When the electrodeposition voltage increased to $12 \mathrm{~V}$, the photocatalytic degradation rate of 


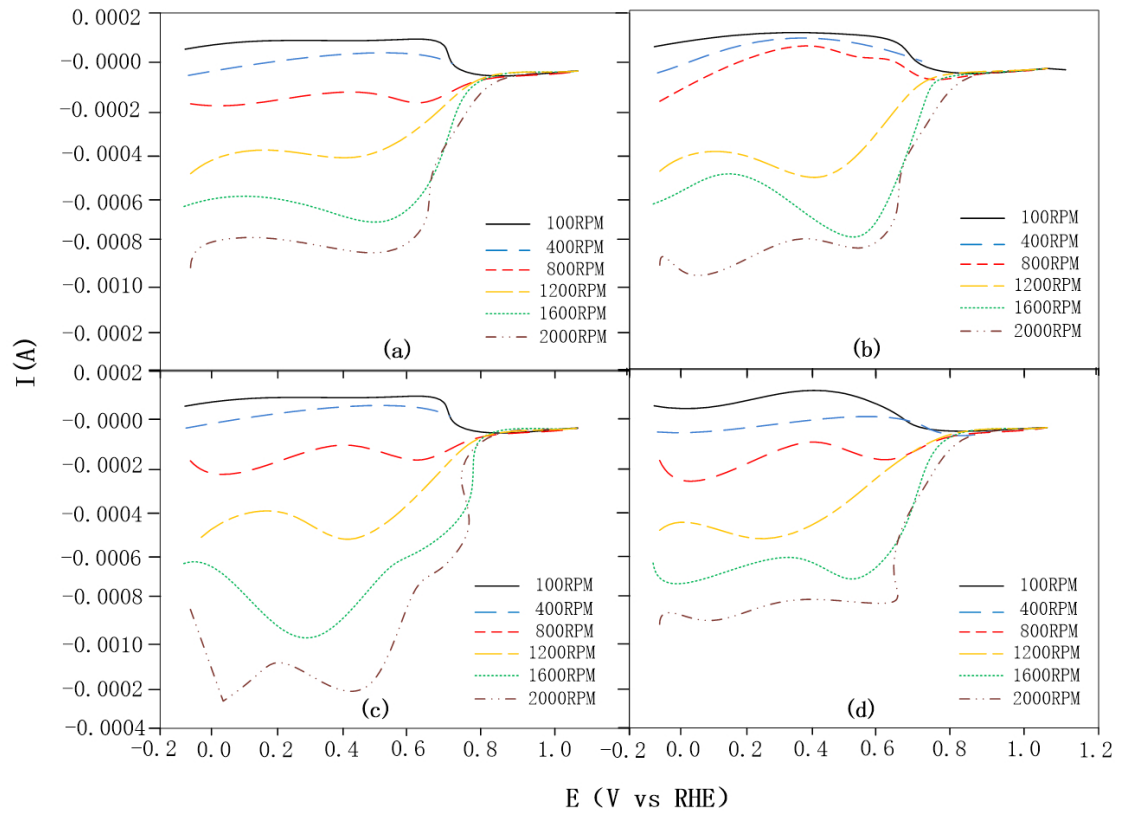

Figure 1: Voltammograms of the electrical stage of gold rings under different samples

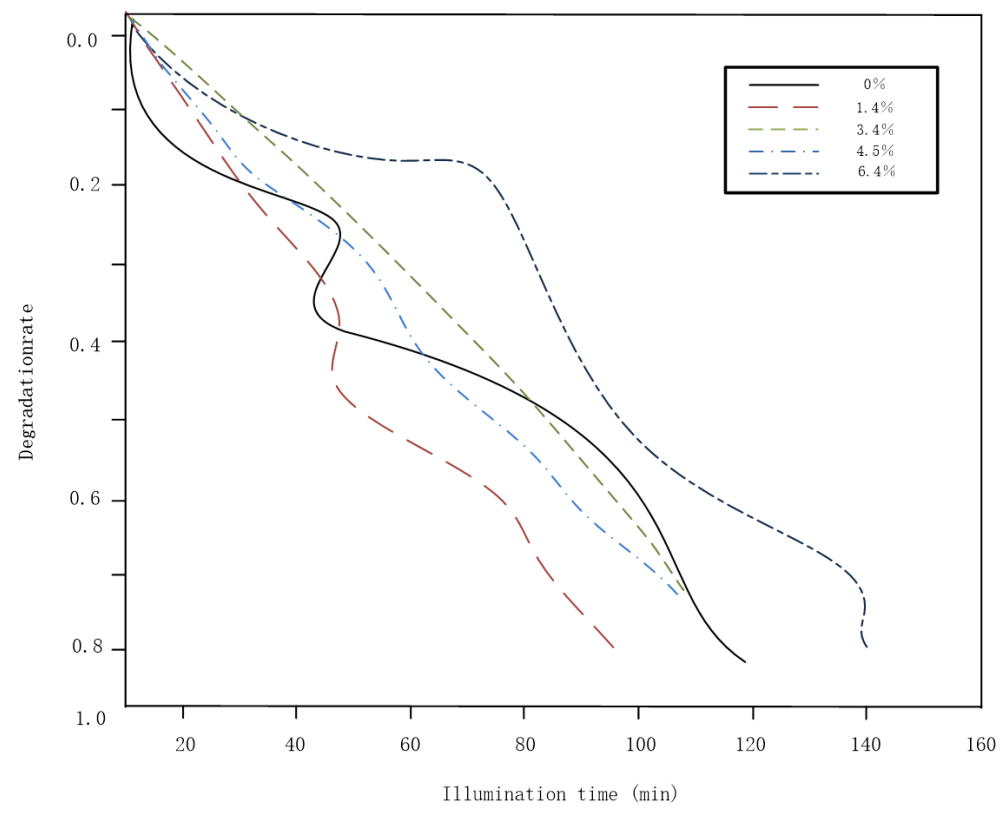

Figure 2: Photocatalytic degradation rate of $\mathrm{Au}-\mathrm{TiO} 2$ nanomaterial patches

the specimens no longer increased with the increase of concentration [5].It is found that the formation of step like band structure with other semiconductors can improve the photocatalytic performance to some extent, and this step like band structure can extend the absorption edge band into the visible region and can reduce the probability of photogenerated electron hole recombination. In previous studies, a large amount of research has focused on the composites of binary semiconductor metal oxides like $\mathrm{TiO} 2 / \mathrm{ZnO}$ 
and $\mathrm{TiO} 2$ / CDs, and it has also been confirmed that such binary semiconductor composites indeed show improved photocatalytic efficiency in the purification of water and the degradation of organic pollutants, and this improvement in photocatalytic efficiency is mainly attributed to the potential gradient across the interfaces, The nanocomposites with different parameters were developed photocatalytic degradation rate test and the results are shown in Figure 2

From the results of the photocatalytic degradation rate test on the top 2 nanomaterial patch, we can see that the $\mathrm{Au}-\mathrm{TiO} 2$ nanocomposite shows the greatest photocatalytic activity when $\mathrm{x}=3.4 \%$ and $4.5 \%$, and the methylene blue solution can be completely degraded after 70 min UV light irradiation. The order of photocatalytic activity of nanocomposites with different Au loadings was $\mathrm{Au}-\mathrm{TiO} 2(4.5 \%)>\mathrm{Au}-\mathrm{TiO} 2(3.4 \%)>\mathrm{Au}-\mathrm{TiO} 2(1.4 \%)>\mathrm{Au}-\mathrm{TiO} 2(6.4 \%)$ $>\mathrm{TiO} 2$.As can be seen from the ex face photocatalysis and electrocatalysis results, the best catalytically active sample all appears at $\mathrm{x}=4.5 \%$.It can be seen from the figure that the content of $\mathrm{Oh}$ increased roughly with the increase of Au loading. This indicates that $\mathrm{Au}$ NPs play a promoting role during the formation of surface oh. From the previous results of photocatalysis and electrocatalysis, it is known that the most catalytically active sample appears at $\mathrm{x}=$ $4.5 \%$, indicating that too much $\cdot \mathrm{Oh}$ may block $\mathrm{O} 2$ adsorption on the catalyst surface and thus reduce the catalytic activity. The Au-TiO2 NPs exhibited obvious catalytic activity in the oxygen reduction reaction of alkaline solution. Meanwhile, compared with TiO2 NPs alone, the addition of Au NPs significantly increased the oxygen reduction activity of the samples: the $\mathrm{n}$ value increased from 2.5 to 3.7 , and the anodic open circuit potential increased from $+0.70 \mathrm{~V}$ to $+0.88 \mathrm{v}$. Among a series of samples, the one with the $\mathrm{Au} / \mathrm{Ti}$ atomic ratio of $4.5 \%$ exhibited the best electrocatalytic performance.

\section{CONCLUSION}

In this paper, a nanomaterial patch is synthesized by microbial technology, which reduces the process cost to some extent and can guarantee green and nontoxic. Microbial preparation of TiO2 nanotubes as well as nanopatch particles were used to study the application of microbial technology in nanomaterial patches, their photocatalytic properties as well as electrocatalytic properties. The experimental results show that the obtained $\mathrm{TiO} 2$ nanotube arrays have the best photocatalytic performance under the examined condition $\mathrm{Au}-\mathrm{TiO} 2$ (4.5\%).When the electrode potential was scanned to $+0.8 \mathrm{~V}$, the cathodic current began to appear in all samples, and the current began to stabilize when the electrode potential reached to $+0.4 \mathrm{~V}$, which illustrated that $\mathrm{Au}-\mathrm{TiO} 2$ nanocomposites had an obvious electrocatalytic activity in oxygen reduction. There are still many deficiencies in the research in the text, and the difficult problems of microorganisms in the application of nanomaterials will be gradually solved in the future, to provide a reliable reference for this research.

\section{REFERENCES}

[1] Lei Zhang. Application of microbial immobilization technology to wastewater treatment [J]. Agriculture and technology, 2021, 41 (14): 139-141

[2] Van Wenhan, Liang Xuesong, and Yiping Luo, et al.exploration of the application of a 3D intestinal microbiota teaching system based on webgl technology for the standardized training of residents [J]. China medical education technology, 2021, 35 (4): 453-457

[3] Cai Anrong. Technological progress of sludge in situ reduction based on microbial metabolic process [J]. Contemporary chemical research, 2021 (14): 155-156

[4] Rongrong Lu, Yan Wang, Yao Huang, et al.. a critical analysis of food microbial detection technology and its quality control [J]. Food circles, 2021 (7): 130-131

[5] Xiao Liu, Hongbing Liu, Chunhua Li. Advances in microbiology of chronic sinusitis using 16SrRNA Gene Sequencing [J]. Journal of clinical Otolaryngology Head \& Neck Surgery, 2021, 35 (7): 658-661 